\title{
Les sociétés jbala et la nature
}

Parlers et relations à autrui dans le Rif, nord du Maroc

Yildiz Aumeeruddy-Thomas, Dominique Caubet, Younes Hmimsa et Ángeles Vicente

\section{(2) OpenEdition}

\section{Journals}

Édition électronique

URL : https://journals.openedition.org/ethnoecologie/3234

DOI : $10.4000 /$ ethnoecologie.3234

ISSN : 2267-2419

Éditeur

Laboratoire Éco-anthropologie

Référence électronique

Yildiz Aumeeruddy-Thomas, Dominique Caubet, Younes Hmimsa et Ángeles Vicente, «Les sociétés jbala et la nature », Revue d'ethnoécologie [En ligne], Supplément 1 | 2017, mis en ligne le 17 octobre 2017, consulté le 19 novembre 2021. URL : http://journals.openedition.org/ethnoecologie/3234 ; DOI : https://doi.org/10.4000/ethnoecologie.3234

Ce document a été généré automatiquement le 19 novembre 2021.

\section{(†) $९$

Revue d'ethnoécologie est mis à disposition selon les termes de la licence Creative Commons Attribution - Pas d'Utilisation Commerciale - Pas de Modification 4.0 International. 


\section{Les sociétés jbala et la nature}

Parlers et relations à autrui dans le Rif, nord du Maroc ${ }^{1}$

Yildiz Aumeeruddy-Thomas, Dominique Caubet, Younes Hmimsa et Ángeles Vicente

\section{Introduction}

1 Ce numéro spécial émane du Projet International de Coopération Scientifique (PICS) France-Maroc (CNRS-CNRST) intitulé « La montagne et ses savoirs (Jbala et Ghomara du Rif occidental, Maroc)». Deux colloques scientifiques, le premier intitulé «Lenguas, memorias y lecturas de la alteridad: Jbala, Ghomara y Rif (Norte de Marruecos) » organisé en Septembre 2014 par Ángeles Vicente, Montserrat Benítez Fernández, Araceli González-Vázquez et Amina Naciri-Azzouz à l'Université de Saragosse, en Espagne, et le deuxième, intitulé "La Montagne et ses Savoirs. Recherches Interdisciplinaires sur le Pays Jbala» organisé par Fouad Brigui, Salama El Fatehi, Younes Hmimsa, Hamid Lahmer, Michel Nieto, Yildiz Aumeeruddy-Thomas et Ángeles Vicente, en novembre 2015 à la Faculté Polydisciplinaire de Larache (Université Abdelmalek Essaâdi) au Maroc, ont donné lieu à des contributions dont une sélection est présentée dans ce numéro.

2 Ce projet a pour objectif principal, grâce à une approche croisée mettant en jeu ethnobotanique, linguistique et anthropologie, de définir les spécificités des groupes sociaux Jbala, Ghomara ainsi que d'autres groupes du Rif, tous ayant en commun des parlers arabes de type montagnard et des pratiques spécifiques sur la nature. Nous nous intéressons tout particulièrement à la façon dont ils tissent des relations à la nature (au sens large) comprenant les plantes, les animaux sauvages et domestiqués, les forêts, la mer et ses éléments, les sols et les micro-organismes ainsi que la surnature, dont en particulier les jnûn, êtres intangibles intégrés de diverses façons dans la vie des habitants du Maghreb et du monde musulman de façon plus vaste.

3 Nous nous référons aussi à des éléments non tangibles d'ordre divin reliant les hommes à la nature couramment désignés sous le terme de baraka. Celle-ci en outre, est un des 
déterminants de l'organisation sociale de certains groupes rifains comme l'a montré Jamous dans ses travaux chez les Iqua'rien du Rif Oriental (2003).

4 Nous examinons la façon dont les savoirs et les savoir-faire, les représentations symboliques de la nature et de la surnature participent à la composition de mondes humains et non-humains intimement liés (Descola 2014). Nous montrons comment ces nombreuses interactions contribuent collectivement à la fabrication de l'histoire de cette région, à la culture, aux parlers, aux rapports à la terre, au territoire et à autrui.

5 Nous avons adopté plusieurs types de notation de l'arabe marocain, selon que les textes étaient relevés, ou non, par des linguistes.

6 1. Les linguistes ont transcrit les textes des parlers arabes de la région étudiée avec une transcription phonologique-phonétique, c'est à dire sans considérer toujours les variantes caractéristiques des variétés de chaque région. Par contre, quand des réalisations fines sont considérées, nous avons eu recours à une transcription phonétique large. Il s'agit du type de transcription couramment utilisé pour les études de dialectologie marocaine. Les correspondances entre les symboles utilisés dans cet ouvrage et ceux de l'alphabet phonétique international, sont les suivantes :

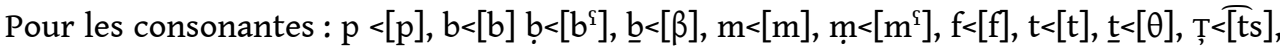

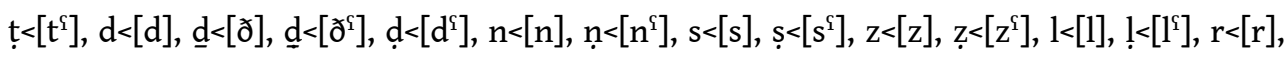

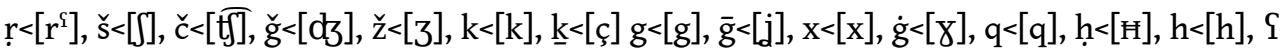
ou $\varepsilon<[\mathrm{C}], \mathrm{P}<[\mathrm{P}], ?{ }^{2}<\left[\mathrm{P}^{\mathrm{S}}\right], \mathrm{w}<[\mathrm{w}], \mathrm{y}<[\mathrm{j}]$.

Pour les voyelles : $\bar{a}<[a:], \bar{i}<[i:], \bar{u}<[u:], \bar{e}<[e:], \bar{o}<[o:], \partial<[ə], a<[a], i<[i], u<[u]$.

9 Par contre, certains ethnobotanistes, notamment Clochey \& Aumeeruddy-Thomas, ont utilisé une écriture adoptée désormais par tous les Marocains dans le langage SMS et sur internet qui permet de noter le $\varepsilon$ transcrit avec un 3 et le $\tau$ transcrit par un 7. Le choix d'utiliser cette écriture est lié au fait que ces termes relevés par les ethnobotanistes n'ont pas tous été enregistrés. Ils ne peuvent donc être réécoutés par les linguistes, et de ce fait ne sont pas transcrits en transcription phonétique.

Enfin, certains mots arabes rentrés dans le langage courant comme jnûn, ou les ethnonymes et les toponymes sont écrits selon une convention orthographique qui est la plus couramment utilisée.

\section{Pourquoi le nord du Maroc et le Rif ?}

11 Le Rif est la région du nord du Maroc qui est intrinsèquement liée à la chaîne de montagne surnommée Rif qui s'étire le long de la Méditerranée et qui forme avec la chaîne de montagne du Sud de l'Andalousie (les cordillères Bétiques), un même arc montagneux se prolongeant de part et d'autre du détroit de Gibraltar. Cette partie de la Méditerranée est celle où les rives nord et sud sont les plus proches sur le plan géologique, géographique, historique, culturel, et linguistique, comme nous le verrons. Le Rif définit deux versants, au Nord, la zone couramment appelée Rif, faisant face à la mer Méditerranée, caractérisée par un littoral riche en petits ports de pêche ainsi que de grandes régions portuaires anciennes et contemporaines tel que Bades associé à l'ancien Royaume médiéval de Nekkor à l'est (Cressier 1983), et Tanger à l'ouest, devenu aujourd'hui le plus grand port de Méditerranée. Le Rif central est situé autour de la ville de Chefchaouen; à l'est, le Rif oriental s'étire jusqu'à Oujda vers l'Algérie avec la ville d'El Hoceima et sur le versant sud, le Pré-Rif est tourné vers les villes de 
Taounate, Ouezzane et Fès ainsi que les plaines céréalières du Gharb. Des reliefs montagneux abrupts et la proximité de la mer en font la région ayant le plus fort taux de pluviométrie du Maroc (Figure 1). Les moyennes annuelles des précipitations sont en effet assez élevées (> $700 \mathrm{~mm}$ ) dans la partie occidentale et dans certaines régions du Pré-Rif, elles atteignent $930 \mathrm{~mm}$ à Melloussa et $1123 \mathrm{~mm}$ à Zoumi (Laouina 1998). Ceci constitue une des particularités de cette région (Maurer 1968), ainsi que la présence d'une végétation forestière remarquable. Contrairement à la rive Nord qui a subi des déforestations importantes depuis le néolithique, les impacts de l'homme sur la végétation démarrent dans le Rif tardivement pendant la période Saâdienne au $\mathrm{XVI}^{\mathrm{e}}$ siècle (Muller et al. 2015). De ce fait et compte tenu des contextes orographique, écologique et humain, le Rif est reconnu comme un « hotspot » de biodiversité pour la région méditerranéenne ainsi qu'à l'échelle globale (Médail \& Quézel 1997).

Figure 1 : Carte situant la chaîne montagneuse du Rif à l'échelle du Maroc

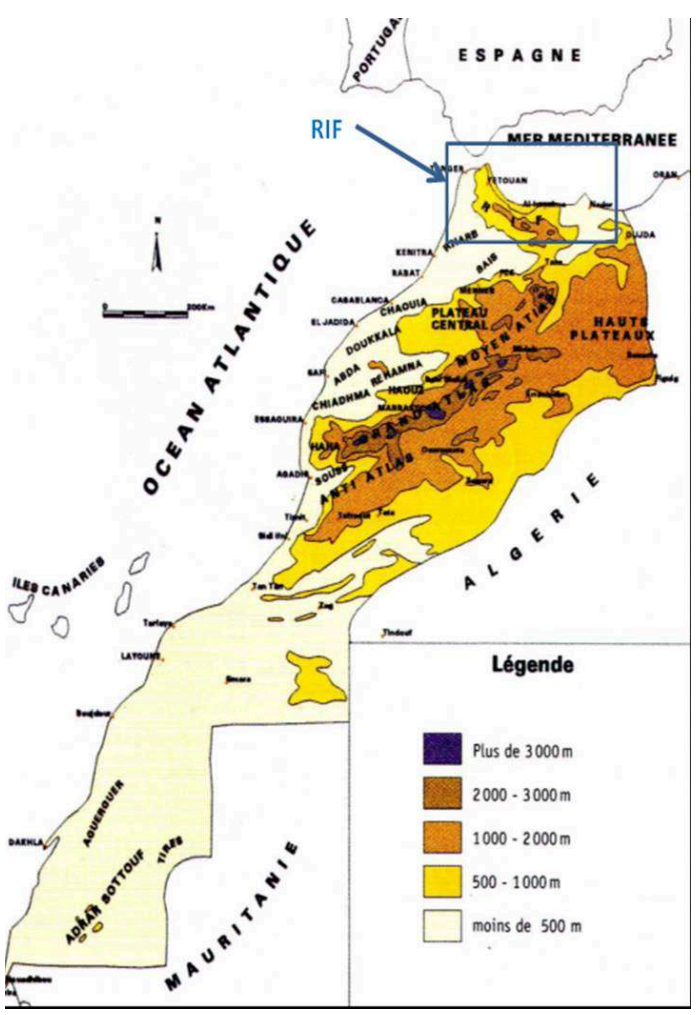

Extrait de Benabid (2000)

Des installations humaines existent dans le Rif depuis le néolithique. Cette zone a connu une culture agraire et alimentaire (fabrication de poteries et de diverses formes de stockage) (Ballouche \& Marinval 2003, González-Vázquez 2014 et Martínez-Sánchez et al. 2017) qui ont inspiré divers travaux en archéobotanique, en anthropologie et en ethnobotanique. En effet, les céréales et en particulier le petit épeautre et l'amidonnier (Triticum monococcum/dicoccum), le blé dur (Triticum durum, Triticum aestivum/durum), l'orge (Hordeum vulgare, Hordeum vulgare var. nudum) ainsi que les légumineuses telles que les lentilles (Lens culinaris), le petit pois (Pisum sativum) et la fève (Vicia faba) étaient présents ainsi que l'élevage d'animaux domestiques et la fabrication de poteries, 5500 à 5000 avant J.-C. (Morales et al. 2016). Les travaux d'ethnobotanique contemporaine menés par les archéobotanistes montrent les techniques de fabrication des récipients 
alimentaires en argile ainsi que les modalités de stockage des céréales en pays Jbala (Peña-Chocarro et al. 2015). Hmimsa \& Ater $(2008,2010)$ ont effectué une étude de l'agrobiodiversité (diversité des espèces de plantes et animaux domestiques ainsi que toute la biodiversité associée) rifaine et décrivent cette région comme un "hotspot " d'agrobiodiversité au Maroc. Ce sont les premières études mettant en relation diversité agraire, terminologies et appellations locales des espèces cultivées du Rif. Les études reliant les terminologies locales et la diversité des figuiers effectuées par Hmimsa et al. $(2012,2017)$ et Aumeeruddy-Thomas et al. (2014) mettent en lumière la richesse du vocabulaire agraire des paysans Jbala concernant les variétés et la désignation des espaces agraires. Divers travaux sur le figuier et l'olivier suggèrent l'importance de cette région dans les processus de domestication pour la région Ouest méditerranéenne (Khadari et al. 2008, Achtak 2009, Aumeeruddy-Thomas et al. 2017).

13 L'histoire, les parlers et l'organisation agraire et sociale de cette région habitée anciennement comme l'ensemble du Maroc par des sociétés berbères, a en outre été influencée depuis la préhistoire par les Phéniciens, les Vandales, les Romains puis les Arabes. Concernant les Arabes, les textes de Ibn Khaldoun (Ayache 1983) et de Camps, historien de la Bérbérie (1983), nous donnent des informations importantes sur le processus d'arabisation des berbères. Située à l'extrême ouest de la Méditerranée comme l'Espagne et le Portugal, le Rif a connu les premières vagues de migration arabes, dites pré-hilaliennes (Marçais 1961), au viI ${ }^{\mathrm{e}}$ siècle, où elles sont arrivées semblet-il par voie terrestre. Elles trouvèrent là un substrat social et linguistique particulier, celui des Berbères du Rif, lui-même composite et fait de plusieurs influences (phéniciennes, latines) qui sont encore visibles dans les parlers Ghomara (Mourigh 2016) et berbères rifains (Kossmann 2000, Lafkioui 2007a et b).

Les parlers arabes Jbala font partie de ce que l'on considère comme la première couche d'arabisation du Maghreb, plusieurs siècles avant l'arrivée des Bédouins, ces derniers arrivant par les hauts plateaux et par le Nord du Sahara, d'abord au XI siècle, puis aux $\mathrm{XIV}^{\mathrm{e}}$ et XVI ${ }^{\mathrm{e}}$ siècles.

En effet, le point de départ de l'arabisation de tout le nord de l'Afrique, c'est la citémère de Kairouan en Ifriqiya (Tunisie) qui essaimera plus tard en créant les foyers de Fès, Tlemcen, Constantine, etc. (Figure 2).

Figure 2 : Carte situant les cités de Kairouan, Tlemcen, Fès ainsi que d'autres sites importants de la période médiévale

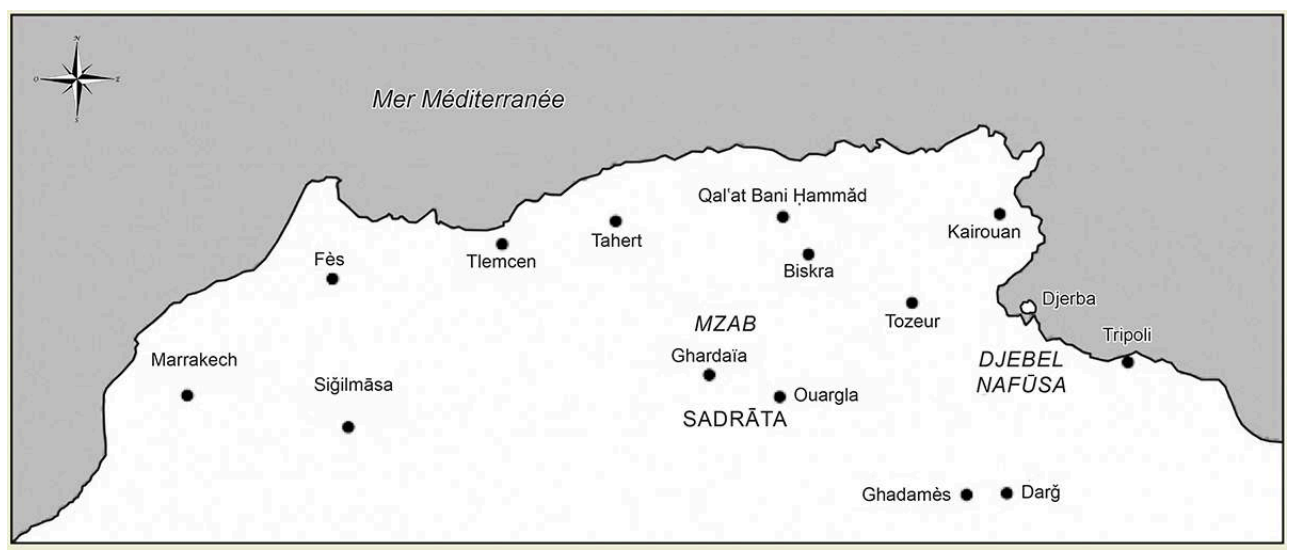

Extrait de Prévost (2008) 

Tlemcen, Constantine et la mer, au nord), vont avoir une grande influence, grâce à leur prestige ; les ruraux qui y habitaient adoptent la langue de la ville, l'arabe, sans pour autant perdre la leur, le berbère (amazighe). Il en résultera un bilinguisme et des contacts qui marquent fortement les deux langues.

Ce sont les formes d'arabe les plus anciennement implantées au Maroc, dits " montagnard » et "villageois » selon la terminologie des dialectologues du début du XXe siècle (Colin 1937, Marçais \& Guîga 1925), avec des archaïsmes, mais aussi des traits innovants, du fait des contacts prolongés avec le berbère ainsi que des nombreux échanges arabo-andalous. Les parlers Jbala, parvenus jusqu'à nous malgré leur spécificité qui en fait parfois des objets de moquerie, ont adopté des traits phonétiques, morphosyntaxiques et lexicaux qui leur donnent un aspect très particulier. Lorsqu'ils ont été documentés au début $\mathrm{du} \mathrm{xx}^{\mathrm{e}}$ siècle, ils ont permis aux linguistes de l'époque, de résoudre l'énigme de certaines étymologies incertaines. Il n'est pas rare que ces parlers aient joué le rôle du "chaînon manquant » dans les essais de reconstructions de formes. De nombreux traits relevés dans les années 1920 sont encore vivants, si l'on en juge par les travaux du début des années 90 et par les résultats des enquêtes récentes (pour un état des lieux sur les parlers arabes des Jbala, (González-Vázquez 2017, Vicente 2017) ${ }^{2}$. La carte présentée dans la figure 3 montre la distribution des différentes situations langagières du Nord du Maroc ainsi que la grande densité des tribus de cette région dont l'organisation segmentaire différencie des ensembles de villages appartenant chacun à une tribu portant le plus souvent un patronyme, tel que Bni Ahmed et liée à une organisation territoriale définie, devenue ultérieurement une commune dont les limites sont toujours présentes.

Figure 3 : Carte extraite de Vignet-Zunz (2014), recomposée afin de mettre en avant les grandes zones linguistiques du Rif et la multitude de frontières séparant les tribus/communes

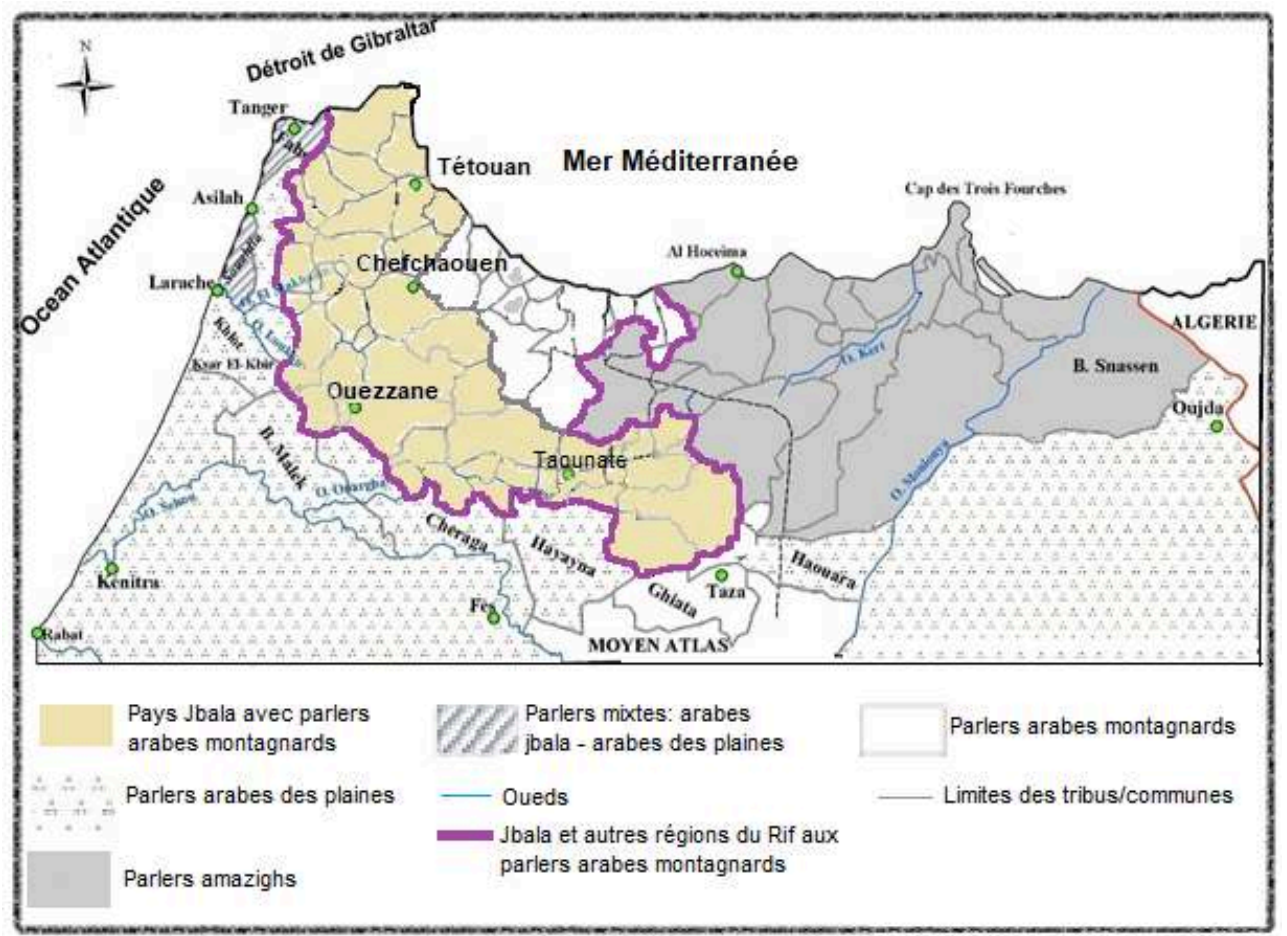

Revue d'ethnoécologie, Supplément 1 | 2017 

étymologie pour essayer de discerner quelques processus de classification de la nature. Ce faisant, les linguistes ont essayé pour leur part de détecter ces traits de dialectologie qui leur sont indispensables pour saisir la diversité des variétés linguistiques en jeu d'un village à l'autre, dans les zones de contacts entre berbérophones et arabophones ou entre différentes zones arabophones parfois d'origines historiques différentes telles que les zones de contacts entre parler arabe Jbala et parler arabe de groupes d'origine bédouine.

\section{Les hommes et la nature dans le Rif}

Plusieurs thèmes émergent de nos analyses et tournent autour de diversités croisées linguistiques, techniques et sociales : 1) Les échanges aux frontières linguistiques et la transmission des savoirs et savoir-faire; 2) Les pratiques, les techniques et leurs nombreuses variantes ; 3) La proximité entre les hommes et les autres : biodiversité et êtres intangibles. 


\section{Les échanges aux frontières linguistiques et la transmission des savoirs et savoir-faire} présentent quelques traits linguistiques particuliers qui leur donnent des identités langagières par rapport à d'autres régions du Maroc. Cette situation, et probablement l'orographie de la zone, ont permis de sauvegarder des traits linguistiques particuliers et caractéristiques du Rif, même si la région n'est pas homogène. On trouve une certaine variation entre les parlers arabes, due à une arabisation plus tardive du sud par rapport au nord de la région examinée ici comme le montre l'article de Barontini \& Hmimsa dans ce numéro sur les différences entre le nord, Anjra, et le sud, Bellota. En même temps, certaines études indiquent qu'outre la variété ghomari, on trouve un autre parler berbère dans la haute montagne de la région de Ketama, parlé par dix tribus. Il s'agit du parler des Senhaja de Sraïr, qui, comme le ghomari, n'est pas Zénète. Tous deux se distinguent du berbère rifain, qui lui, est considéré comme de type Zénète (Kossmann 2017). langagière par rapport aux parlers arabes des villes septentrionales voisines. Ceci constitue encore une frontière poreuse où les situations de variation linguistique émanant du contact entre les parlers Jbala et les parlers citadins, sont nombreuses.

Ces situations sont illustrées dans diverses études de ce numéro qui montrent que les pratiques et les ressources traversent ces frontières langagières poreuses. Les contacts entre la population de différentes communautés linguistiques existent grâce aux échanges aux souks de la région et aux mariages. Ainsi, à la frontière entre Jbala et 
Hyayna, communauté d'origine bédouine voisins de la communauté Jbala d'Aïn Mediouna, Aumeeruddy-Thomas \& Caubet (ce numéro), ont identifié des parlers, ainsi que des traits culturels clairement différenciés. Cependant, ces deux communautés partagent des techniques agraires et apicoles, ont des échanges économiques au souk ainsi que des échanges matrimoniaux, bien que ces derniers soient probablement plus rares qu'au sein des deux groupes. Ces travaux mettent en évidence, en outre, le rôle des femmes qui doivent quitter leur village pour rejoindre celui de leurs époux, dans la circulation de techniques et pratiques domestiques.

Les changements socio-économiques des dernières années ont provoqué une plus grande interaction entre les habitants de différentes zones et par conséquent, un plus grand nombre de situations de contact linguistique. Le résultat est une variabilité linguistique dans les différents parlers avec l'effet final d'un nivellement des traits considérés comme plus locaux ou anciens surtout chez les plus jeunes comme le montrent Naciri-Azzouz \& Vicente (ce numéro).

D'autres types de frontières sont mis en lumière dans ce numéro. Ainsi, l'article de Delplancke et Aumeeruddy-Thomas montre l'importance de la différenciation entre ce qui appartient au territoire (bəldi), façonné par des techniques et des pratiques locales de reproduction des plantes, dans ce cas précis l'amandier, par opposition au matériel et aux techniques étrangères (rōomi). Entre bəldi et rōomi, la porosité biologique passe par la transformation des rōmi en boldi par des ensembles de pratiques favorisant la reproduction sexuée spontanée des graines issues des amandiers rōmi. La reproduction sexuée des amandiers est la technique locale privilégiée alors que les rōmi sont habituellement multipliée par greffage, une technique venue d'ailleurs et autrefois imposée par les Espagnols. Ainsi le rōomi se refond dans le baldi par le biais de pratiques accompagnants la reproduction des arbres.

Parmi les frontières les plus subtiles, celles se situant entre le tangible et l'intangible sont illustrées par le papier de González-Vázquez (ce numéro) qui démontre remarquablement grâce à l'analyse d'une série de scénettes, comment se jouent les pratiques de l'hospitalité entre le monde des hommes et celui des jnûn, deux mondes qui sont symboliquement distincts. Cette contribution montre des pratiques alimentaires qui symbolisent l'hospitalité et qui établissent des ponts entre le tangible et l'intangible. La porosité entre les hommes et les jnûn intervient quand l'intériorité des hommes est habitée par ces derniers, instaurant une continuité entre hommes et jnûn par-delà cette frontière entre tangible et intangible avec de nombreuses conséquences sociales, sur la santé et relationnelles.

Enfin la contribution de Clochey et Aumeeruddy-Thomas (ce numéro) montre une toute autre frontière, celle qui sépare les plantes cultivées et les espèces adventices des cultures qui poussent spontanément. En effet, bien que les plantes adventices soient totalement intégrées aux zones cultivées ou aux jachères et ont probablement évolué au cours du temps aux côtés des céréales et des légumineuses alimentaires, l'étymologie des noms de ces herbes montre une part importante de termes associés à des animaux non-domestiqués (vipère, scorpion etc.). Ceci les distingue des terminologies des plantes cultivées qui font référence dans certains cas à des animaux domestiques (Hmimsa et al. 2012). Elles représentent aussi pour beaucoup d'entre elles une ressource alimentaire de grande qualité, bien qu'accessible uniquement aux périodes printanières. Nous avons ainsi typiquement ici deux ensembles de plantes qui 
se côtoient dans les espaces agraires et dont le traitement fait appel à des catégories linguistiques et des usages bien distincts.

\section{Les pratiques, les techniques et leurs nombreuses variantes}

Diverses contributions de ce numéro mettent en exergue des ensembles de pratiques et de techniques liés à l'usage des plantes, l'élevage des animaux, ou sur les productions. Ces techniques montrent des liens entre savoirs ethnoécologiques sur la phénologie, l'écologie des espèces et les types de productions recherchées. Des techniques de postproduction multiples, fabrication de farines diverses, de pain, extraction d'huile, montrent que ces modalités de transformation des produits contribuent aux identités culturelles, une situation courante des systèmes d'alimentation à travers le monde (Barrau 1983). La diversité des techniques et des pratiques semble bien correspondre à la diversité culturelle du Rif (diversité de langues et d'origines des groupes sociaux en présence). Cette diversité est associée à l'histoire des interactions avec le monde méditerranéen au fil de périodes de l'histoire distinctes et de diverses formes de rencontre entre groupes berbérophones et arabophones, mais aussi comme c'est évoqué plus haut la diversité orographique de ces montagnes.

\section{Liens entre phénologie des plantes et pratiques techniques}

Zambu, la farine d'orge jeune séchée/grillée au four est produite avec des graines d'orge à peine arrivées à maturité. Cette pratique prend en compte la phénologie des céréales et les besoins des hommes car il s'agit d'une période de soudure (Aumeeruddy-Thomas \& Caubet ce numéro). Elle se réfère à des pratiques semblables dans l'ensemble du monde Méditerranéen et au Maroc selon d'autres appellations (səllu ou zəmmēta). Cette farine, et les façons de l'utiliser ont une très grande valeur culturelle et relationnelle. Un usage semblable d'olives non-matures grillées/séchées au four donnant une huile spéciale, Eolwāna, est décrit par Aumeeruddy-Thomas et Caubet (ce numéro). Dans ces deux situations il s'agit de dispositifs techniques complexes mettant en jeu des objets domestiques avec diverses variantes d'une région à l'autre y compris sur les appellations. Par exemple farrān d-əz-zənqa, forna et aynur pour désigner le four domestique, gəșEa, tažəfnit pour désigner les plats en bois ou en terre cuite, utilisées pour pétrir le pain ainsi que pour " pétrir » les olives, l'usage d'une meule élémentaire (pierre plate et pierre ronde à va et vient) et ou d'une petite presse mobile, de tissus divers (dərra d-əl hayāti, sințit) etc. Ces deux ensembles techniques montrent une résilience et une stabilité remarquable de pratiques et de savoirs typiquement féminins, probablement très anciens, révélés ici par les enquêtes et les comparaisons d'appellations et de termes linguistiques. La grande diversité d'huiles reconnues et utilisées dans diverses circonstances nous semble être fortement associée aux agroécosystèmes à oléastre greffés par des variétés d'oliviers, qui sont caractéristiques du pays Jbala (Aumeeruddy-Thomas et al. 2017). De même le lexique associé aux pratiques d'élevage par Naciri-Azzouz et Vicente (ce numéro) montre l'étendue des savoirs féminins et la proximité de leurs vies avec celle des animaux. Les changements de mode de vie en cours, exode rural très marqué et modernisation agricole, tendent à fragiliser ces pratiques ainsi que les parlers, vocabulaires et mode de vie associés. La transmission de ces techniques à travers l'ensemble du pays Jbala incombe en partie aux femmes comme nous le montrons à travers l'analyse des échanges matrimoniaux, 
mais également aux transactions des hommes au sein des souks (Caubet \& Aumeeruddy-Thomas ce numéro).

\section{Multiplication sexuée versus multiplication végétative} nettement de concepts relatifs à la domestication des arbres en Méditerranée qui mettent en exergue l'importance de la reproduction végétative comme moteur principal de la domestication (Zohary et al. 2012). Parmi les points clés relevés, nous montrons dans diverses contributions une gestion des arbres issus de graines, se développant par multiplication sexuée chez les trois espèces arborées emblématiques du Rif (et de la région méditerranéenne), mettant en jeu des dynamiques en même temps très différentes. Il s'agit d'arbres épars se multipliant spontanément chez le figuier, qui sont conservés et nommés, de populations entières d'amandiers multipliés par graines, poussant spontanément ou semés dans les champs, et de vastes populations d'oléastres. Ces derniers proviennent de la forêt nettoyée où ils ont été conservés. Les catégories nommées qui se multiplient par voie sexuée sont bien identifiées tel que nābūt chez le figuier (Hmimsa, Aumeeruddy-Thomas \& Ater, ce numéro) l-barri chez l'olivier (Aumeeruddy-Thomas \& Caubet, ce numéro) et l-lūz bəldi chez l'amandier (Delplancke et Aumeeruddy-Thomas, ce numéro). Dans le cas du figuier, il s'agit d'une dynamique pouvant entraîner l'incorporation de nouvelles variétés. Pour l'olivier, la gestion des oléastres est destinée à l'huile mais également à obtenir des porte greffes résistants dans un environnement hétérogène. Concernant l'amandier, il s'agit du mode de gestion le mieux adapté aux contextes agrosylvopastoraux des régions plus sèches de l'est du Rif (Delplancke 2011). Figuiers, oliviers et amandiers sont par ailleurs aussi multipliés végétativement par bouture, ou par greffage, des techniques qui sont parfaitement maîtrisées localement par les paysans.

Ces différents cas montrent que les catégories nommées permettent de mettre en lumière des techniques peu connues à l'échelle méditerranéenne, qui sont des portes d'entrée sur la compréhension des processus de diversification variétale et de domestication de ces trois arbres emblématiques. L'importance de la baraka divine associée à ce qui pousse sans l'aide des hommes est aussi à considérer dans les relations que les hommes lient avec cette part non contrôlée du domaine agraire.

\section{La proximité entre les hommes et les autres : biodiversité et êtres intangibles}

La proximité est prise ici dans son sens très courant, c'est-à-dire le fait d'être très proche, de vivre ensemble. Nous observons une grande proximité entre les hommes et la diversité des plantes et des animaux domestiqués, non-domestiqués ainsi que des êtres invisibles ou intangibles. Nous montrons également la façon dont cette diversité définit un ensemble de relations sociales: les liens que les hommes et les femmes nouent entre eux à propos de l'agrobiodiversité et avec les êtres intangibles que sont les jnûn. Les formes d'interactions qui fondent cette proximité montrent que la biodiversité et les êtres de la nature au sens large participent activement au collectif des hommes et à la définition de leur identité et la composition de leur monde. Nous retrouvons ici des schèmes régissant les relations entre les hommes et la nature et la 
façon dont ces liens participent à la composition des mondes (Descola 2014). Parallèlement, cette proximité et la diversité des modalités d'interactions qui en découle, semble marginalisée ou en cours de transformation et met en péril par la même occasion la base culturelle de ces groupes sociaux, ainsi que leurs parlers.

Les travaux présentés par Barontini et Hmimsa (ce numéro) mettent en exergue la grande diversité des fruitiers qui repose sur des notions perceptuelles très fines portant sur un lexique se référant à la couleur des fruits, leurs goûts, leur capacité à pousser dans tel ou tel sol. Nous nous trouvons ici dans le cadre très classique de l'hortus (au sens de Barrau 1986), de la pratique du jardinier qui connait précisément toutes les facettes de la diversité qu'il manipule. Cette diversité se retrouve dans les žnān, Garsa ou bhirra, jardins liés aux maisons dans la région de Bni Boufrah plus ou moins associés à des sources d'eau (sources, oued, puits) (Delplancke \& Aumeeruddy-Thomas, ce numéro). Ces terminologies changent d'une région à l'autre dans le Rif. Par exemple, chez les Bni Ahmed, les Yarsa sont toujours associés à des plantations de bord d'oued alors qu'à Ouezzane la Yarsa fait référence aux plantations d'oliviers se trouvant sur les collines.

On retrouve une richesse d'interactions entre les hommes et la diversité des plantes dans les pratiques de cueillette de plantes adventices ramassées au printemps et dont l'analyse de l'étymologie révèle une richesse linguistique indéniable (Clochey \& Aumeeruddy-Thomas, ce numéro). Un mélange fait de nombreuses espèces d'adventices rentre dans la préparation de la baqqūla, plat d'herbes cuites mélangées et dont la valeur culturelle et diététique est très importante. Nonobstant, les travaux sur les cultures marginalisées (El Fatehi \& Ater, ce numéro) ont révélé que malgré l'importance historique et culturelle de certaines plantes domestiquées, le risque de perte de leur proximité est corrélé par la perte de leur usage.

Une très grande proximité quotidienne se retrouve aussi dans les pratiques d'élevage des femmes Ghomara et Jbala, comme illustré dans le lexique présenté par NaciriAzzouz \& Vicente (ce numéro). Toutes les catégories d'animaux connus selon leur maturité, leur gestation, leur capacité à allaiter, fait appel à un lexique élaboré qui selon ces auteurs tend à disparaître au fur et à mesure que les changements de mode de vie (modernisation, exode) mettent fin à ces pratiques de proximité.

Ce travail met l'accent sur un lexique qui peut renseigner sur l'histoire linguistique de la région. La plupart des mots relevés sont d'origine arabe, avec quelques exemples de mots berbère et même d'origine latine/romaine. Il s'agit d'un vocabulaire riche qui montre un certain degré d'homogénéité même s'il y a aussi des variations entre les deux zones étudiées.

Enfin les jnûn font l'objet de différentes formes d'hospitalité comme le montre González-Vázquez. Ceci nous indique le degré de sociabilité et les pratiques élaborées que nécessite le vivre ensemble avec ces êtres extrêmement présents dans le quotidien des habitants. Comme l'indique González-Vázquez, ces pratiques ne sont nullement spécifiques au Rif, mais bien communes à l'ensemble du Maghreb et toujours extrêmement présentes dans cette région. 


\section{Conclusion} nous semblent être à l'origine de toutes ces diversités. En effet ces effets de frontières introduisent des discontinuités productives d'identité ou plutôt, ce sont diverses identités d'origine sociale, historique, et/ou politique, qui les produisent et les maintiennent malgré les porosités. Ces dernières ajoutent à la diversité par l'apport discret, rare ou épisodique de nouveaux éléments sur tous les plans (linguistique, social, biologique etc.). Sur le plan social, l'organisation des tribus et des inter-tribalités tracent des territoires relativement stables (Figure 3). Les Hyayna ne sont pas des Jbala et les Jbala ne sont pas des Berbères etc., mais leurs rencontres respectives ont produit des innovations et de la diversité au cours du temps, par différents phénomènes d'adoption et d'innovation, linguistique, technique, ainsi que d'agrobiodiversité (diversité variétale). Les relations intertribales régissent des relations d'échanges matrimoniaux et d'échanges économiques. Les frontières et porosités politiques sont celles qui ont différencié, puis mis en contact l'Afrique du Nord et le monde romain sans compter les échanges bien plus anciens qui caractérisent la Méditerranée (période phénicienne, carthaginoise etc.). L'arabisation au viI siècle a apporté des normes et de nombreux échanges de langues, de techniques et de relations commerciales émanant du monde musulman. Ceci a permis une unification, suivie d'une dissociation du nord de l'Afrique et d'al-Andalus à l'époque médiévale où les dynasties berbères tel les Almohades ont joué un rôle important. À l'époque contemporaine, les colonisations espagnole et française ont également profondément marqué respectivement, le Rif (espagnol) et le Pré-Rif (français). Aujourd'hui la globalisation va-t-elle annihiler les effets de toutes ces frontières et des échanges qui ont contribué à cette diversité d'identités locales? La question est ouverte et mériterait d'autres travaux interdisciplinaires, utilisant des marqueurs tout autant linguistiques, ethnologiques que biologiques.

en numéro nous amène à considérer que les discontinuités qui séparent des faits du réel vastes et plus ou moins stables (langues, organisation tribale, ressources biologiques), et les continuités introduisant des apports plus discrets, rares et comparativement moins nombreux que les échanges ayant lieu au sein des zones stables. On citera par exemple l'adoption et les échanges linguistiques aux frontières, ou les échanges entre les intériorités des hommes et ceux des jnûn, l'apport de la baraka divine dans les pratiques agraires, ou le transfert de pratiques ou de ressources biologiques aux frontières grâce à des échanges matrimoniaux généralement rares, ou dans les souks. Tout ceci constitue un champ dynamique d'interactions qui produit de la diversité à différents échelles culturelles, sociales, linguistiques, techniques et agrobiologiques.

Ce numéro spécial restitue, grâce aux approches ethnobotaniques, ethnozoologiques, linguistiques et anthropologiques, les modalités d'interactions entre les hommes, les plantes, les animaux et les entités intangibles dans le Rif. Notre approche

Revue d'ethnoécologie, Supplément 1 | 2017 
interdisciplinaire nous permet d'accéder aux faits du réel et au quotidien des hommes, des femmes et des enfants des villages de cette région reculée et assez peu accessible du Maroc. Ceci révèle mille détails rendus intelligibles grâce à des corpus précis et une analyse linguistique fine mise en ligne pour certains, et richement illustrés de photos, qui nous permet d'entrer en contact avec des réalités autres, rarement perceptibles. Les analyses ethnobotaniques et anthropologiques révèlent des versants complémentaires du tissage qui s'est établi au cours du temps en pays Jbala entre les Hommes et Autrui (la nature et les êtres intangibles).

Nous remercions le Programme de Coopération Scientifique (PICS) « La montagne et ses savoirs Etudes sur le pays Jbala (nord-ouest du Maroc) » fondé sur un partenariat entre le CNRS, France et le CNRST, Maroc. Ce programme a été coordonné par l'IREMAM (CNRS, Aix-Marseille Université, sous la direction de Ghislaine Alleaume, puis Catherine Miller) et le Labo-REL de la Faculté des Lettres et des Sciences Humaines, Dhar El Mehraz, Université Sidi Mohammed Ben Abdellah, Fès (sous la direction de Fouad Brigui), entre 2013 et 2015. Nous remercions tout particulièrement Catherine Miller et Michel Nieto de l'IREMAM, qui ont participé à la relecture de ce numéro spécial et pour leur aide afin de mener à bien ce programme. Nous remercions Mohammed Ater de l'Université Abdelmalek Essaâdi, Tétouan, qui a apporté une aide précieuse lors de l'initiation du PICS. Ce projet a été l'occasion de réunir des chercheurs de différentes équipes et disciplines autour d'un réseau tri-national (Maroc-France-Espagne), partageant une réflexion commune autour d'un champ de recherche qui s'intéresse aux variantes linguistiques, à la culture, à la société et à la biodiversité des montagnes rifaines au Maroc. Les partenaires de ce projet, que nous remercions chaleureusement, sont : l'Université Abdelmalek Essaâdi, Tétouan, le Centre Jacques Berque, Rabat, l'Université de Saragosse, le Centre d'Écologie Fonctionnelle et Évolutive UMR 5175, CNRS, Montpellier, le LaCNAD (Langues et Cultures du Nord de l'Afrique et Diasporas) EA 4092, INALCO, Paris et, l'Université Mohammed Premier, Oujda. Ce PICS a aussi travaillé avec des partenaires associatifs tels que le CÉRI-Groupe Jbala ou la Fondation Anna Lindh, qui ont coordonné une série de conférences auxquels les chercheurs ont participé avec d'autres acteurs de la société civile.

\section{BIBLIOGRAPHIE}

Abou El Haja H. 1995 - Habla árabe en duwar. Srema (tribu Bni Qorra, provincia de Taunat). Textos (transcripción, traducción y notas) y estudio fonético y morfológico. Mémoire de Licence d'Espagnol non publié sous la direction de Simon Lévy. Rabat, Université Mohamed V, 1994-1995, 95 p.

Achtak H 2009 - Domestication et diversification variétale chez le figuier au Maroc: bases pour la conservation et la valorisation des ressources génétiques locales. PhD Thesis, Supagro, Montpellier.

Auclair L., Aspe C. \& Baudot P. 2006 (Ed.) - Le retour des paysans? À l'heure du développement durable. Aix en Provence, EDISUD, 358 p.

Aumeeruddy-Thomas Y., Hmimsa Y., Ater M. \& Khadari B. 2014 - Beyond the divide between wild and domesticated: spatiality, domesticity and practices pertaining to fig (Ficus carica L.) and olive (Olea europaea L.) agroecosystems in Morocco. In : Chevalier A., Marinova E., Peña-Chocarro L. 
(Ed.) Crops and people: choices and diversity through time. Brussels, Earth EU, London, OXFAM : 191-197.

Aumeeruddy-Thomas Y., Moukhli A., Haouane H. \& Khadari B. 2017 - Ongoing domestication and diversification in grafted olive-oleaster agroecosystems in Northern Morocco. Regional Environmental Change 17 :1315-1328.

Ayache G. 1983 - bn Khaldoun et les Arabes. In Ibn Khaldoune. Dar al kitab (éd.). In : Étude d'histoire marocaine. (1983). Société Marocaine des Éditeurs Réunis (SEMR-Rabat) : 23-39.

Ballouche A. \& Marinval P. 2003 - Données palynologiques et carpologiques sur la domestication des plantes et l'agriculture dans le Néolithique ancien du Maroc septentrional (site de kaf taht elghar). Archéométrie 27 : 49-54.

Barkat-Defradas M. \& Motte-Florac E. 2016 - Words for odours. Language skills and cultural insights (Ed) Newcasthe upon Tyne, Cambridge Scholars Publishing, 265 p.

Barrau J. 1983 - Les hommes et leurs aliments. Esquisse d'une histoire écologique et ethnologique de l'alimentation humaine, Temps actuels, $378 \mathrm{p}$.

Barrau J. 1986 - Les hommes et les forêts tropicales d'Afrique et d'Amérique. Mémoires du Muséum National d'Histoire Naturelle, Paris MNHN 132 : 289-296.

Behnstedt P. 2002 - La frontera entre el Bereber y el Árabe en el Rif. Estudios de dialectología norteafricana y andalusí $6: 7-18$.

Benabid A. 2000 - Flore et écosystèmes du Maroc: évaluation et préservation de la biodiversité. Paris, Ibis Press, $359 \mathrm{p}$.

Camps G. 1983 - Comment la Berbérie est devenue le Maghreb arabe. Revue de l'Occident musulman et de la Méditerranée 35 :7-24.

Caubet D. 2017 - Les parlers du Nord-Ouest marocain à partir de corpus recueillis dans la région en 1992-1995 (sous la direction de Simon Lévy). In Vicente Á., Caubet D. \& Naciri Azzouz A. Ed.), La région du Nord-Ouest marocain: Parlers et pratiques sociales et culturelles. Zaragoza, Prensas de la Universidad de Zaragoza : 109-142.

Colin G. S. 1921 - Notes sur le parler arabe du nord de la région de Taza. In Bulletin de l'Institut Français d'Archéologie Orientale 18 : 33-119.

Colin G. S. 1937 - Les parlers : l'arabe. Initiation au Maroc. Rabat, Institut des Hautes Etudes Marocaines : 208-236.

Conklin H. 1954 - The relation of Hanunoo culture to the plant world. PhD Dissertation in Anthropology, Yale University, New Haven.

Cressier P. 1983 - Fortifications du Rif. In: Castrum I. Habitats fortifiés et organisation de l'espace en Méditerranée médiévale. Table-ronde tenue à Lyon les 4 et 5 mai 1982. Lyon : Maison de l'Orient et de la Méditerranée Jean Pouilloux, 1983. pp. 45-55. (Travaux de la Maison de l'Orient) http:// www.persee.fr/web/ouvrages/home/prescript/article/mom_0766-0510_1983_act_4_1_1943

Delplancke M 2011 - Histoire évolutive de l'amandier cultivé (Prunus dulcis) en Méditerranée. Regards croisés sur la domestication, dialogue entre la biologie et l'ethnobiologie. Thèse de Doctorat de l'Université Montpellier II, $264 \mathrm{p}$.

Descola P. 2014 - La composition des mondes. Paris, Flammarion, 288 p.

Friedberg C. 1990 - Le Savoir botanique des Bunaq : percevoir et classer dans le Haut Lamaknen (Timor, Indonésie) Paris, Éditions du Muséum 302 p. 
González-Vázquez A. 2014 - La fábrica femenina del barro y de sus rituales : la producción de cerámicas de las mujeres de la cabila Ghzawa (Yebala, Marruecos). In Benítez Fernández M. (Ed.), Trabajo y palabra: dos formas de expresión de las mujeres de Marruecos. Zaragoza, Prensas de la Universidad de Zaragoza : 73-119.

González-Vázquez A. 2017 - Les études linguistiques sur les variétés de l'arabe au nord-ouest du Maroc (1779-1956). Quels savoirs ethnographiques et anthropologiques sur l'Autre et l'Ailleurs véhiculent-elles? In Vicente Á., Caubet D. \& Naciri-Azzouz A. (Ed.), La région du Nord-Ouest marocain : Parlers et pratiques sociales et culturelles. Zaragoza, Prensas de la Universidad de Zaragoza :51-92.

Haudricourt A.G. \& Hédin L. 1943 - L'homme et les plantes cultivées. Paris, Gallimard.

Hmimsa Y. \& Ater M. 2008 - Agrodiversity in the traditional agrosystems of the Rif mountains (north of Morocco). Biodiversity: Journal of life on earth 9 (1-2) : 78-81.

Hmimsa Y. \& Ater M. 2010 - Le savoir-faire paysan : quelques exemples. In : Ministère de la culture (Ed.), Regards sur les patrimoines et les terroirs des Jbala. 3 éme Forum International PlanèteTerroirs, Chefchaouen-Maroc : 73-78.

Hmimsa Y., Aumeeruddy Thomas Y. \& Ater M. 2017 - Lexique sur les figuiers: exemple des variétés nommées chez les Beni Ahmed (Jbala, Rif occidental). In Vicente Á., Caubet D. \& NaciriAzzouz A. (Ed.), La région du nord-ouest marocain : Parlers et pratiques sociales et culturelles. Zaragoza, Prensas de la Universidad de Zaragoza : 265-274.

Hmimsa Y., Aumeruddy Thomas Y. \& Ater M. 2012 - Vernacular taxonomy, classification and varietal diversity of fig (Ficus carica L.) among Jbala cultivators in Northern Morocco. Human Ecology 40 : 301-313.

Jamous R. 2003 - Honneur et Baraka, les structures sociales traditionnelles dans le Rif. Paris, Editions de la Maison des Sciences de l'Homme et Cambridge University Press, $300 \mathrm{p}$.

Khadari B., Ater M., Achtak H., Oukabli A., Roger J.P. \& Kjellberg F. 2008 - Moroccan fig present specific genetic resources: a high potential of local selection. In Leitao J. et Neves M.A. (Ed.), Proceedings of the Third International Symposium on Fig, Acta Horticulturae $N^{\circ} 708: 33-38$.

Khoukh A. 1993 - El habla jebli de la ciudad de Wazzan en 1992. Mémoire de licence d'espagnol non publié sous la direction de Simon Lévy. Rabat, Université Mohamed V 1992-1993, 70 p.

Kossmann M. 2000 - Esquisse grammaticale du rifain oriental. Paris-Louvain, Ed. Peeters, X-215 p.

Kossmann M. 2017 - La place du parler des Senhaja de Sraïr dans la dialectologie berbère. In Vicente Á., Caubet D. \& Naciri Azzouz A. (éds.), La région du Nord-Ouest marocain: Parlers et pratiques sociales et culturelles. Zaragoza, Prensas de la Universidad de Zaragoza : 93-108.

Lafkioui M. 2007 - Atlas linguistique des variétés berbères du Rif, (extracts), Berber Studies, vol. 16. Köln, Rüdiger Köppe Verlag. [En ligne] URL : http://menalafkioui.weebly.com/uploads/ 7/2/9/5/7295665/lafkioui_extract_atlas_2007.pdf. Consulté le 15 juin 2017.

Laouina A. 1998 - L'environnement des montagnes du Nord : Atouts, contraintes et processus de dégradation. In Justus Perthes Verlag Gotha (Ed.), Le Développement du Maroc Septentrional : points de vue de géographes. Allemagne, Nahost und Nordafrika : 15-59.

Lévi-Provençal E. 1922 - Textes arabes de l'Ouargha, dialecte des Jbala (Maroc septentrional). Paris, E. Leroux, $285 \mathrm{p}$. 
Maghdad A. 1993 - El habla árabe en el Aduar de Msek (Textos, Traducciones, Notas y Compendio). Mémoire de licence d'espagnol non publié sous la direction de Simon Lévy, Rabat, Université Mohamed V, 1992-1993, 88 p.

Marçais W. \& Guîga A. 1925 - Textes arabes de Takroûna (Textes, Transcription et Traduction annotée) Tome I. Paris, Imprimerie Nationale, $426 \mathrm{p}$.

Marçais W. 1961 - Comment l'Afrique du Nord a été arabisée. In Articles et Conférences. Paris, A. Maisonneuve : 171-192.

Martínez-Sánchez R., Vera-Rodríguez J. C., Moreno-García M., Perez G., Peña-Chocarro L. \& Bokbot Y. 2017 - Beyond Adornment: Cowry Use as Potter's Tool in the First Impressed Wares of the Southwestern Mediterranean Coast (Northern Morocco). The Journal of Island and Coastal Archaeology, http://dx.doi.org/10.1080/15564894.2017.1284963

Maurer G. 1968 - Les paysans du Haut Rif central. Revue de Géographie du Maroc, N¹4 : 3-70.

Médail F. \& Quézel P. 1997 - Hot-Spots analysis for conservation of plant biodiversity in the mediterranean bassin. Annals of the Missouri Botanical Garden 84 :112-127.

Messaoudi L. 1999 - Étude de la variation dans le parler des Jbala (Nord-Ouest du Maroc). Estudios de dialectología norteafricana y andalusí 4 : 167-176.

Morales J., Pérez Jordá G., Peña-Chocarro L., Bokbot Y., Vera J. C., Martinez Sánchez R. M. \& Linstädter J. 2016 - The introduction of South-Western Asian domesticated plants in NorthWestern Africa: An archaeobotanical contribution from Neolithic Morocco. Quaternary International, 412 : 96-109. [en ligne] URL : http://dx.doi.org/10.1016/j.quaint.2016.01.066. Consulté le 15 juin 2017.

Mourigh Kh. 2016 - A Grammar of Ghomara Berber (North-West Morocco). Köln, Rüdiger Köppe Verlag, $443 \mathrm{p}$.

Muller S.D., Rhazi L., Andrieux B., Bottollier-Curtet M., Fauquette S, Saber E, Rifai N. \& DaoudBouattour A. 2015 - Vegetation history of the western Rif mountains (NW Morocco): origin, lateHolocene dynamics and human impact, 487-501. https://doi.org/10.1007/s00334-014-0504-9

Naciri-Azzouz A. 2016 - Les variétés árabes de Ghomara : s-saḩ̨l vs. əğ-ğbəl (la côte vs. la montagne). In : Grigore G. \& Bituna G. (Ed.). Arabic varieties: Far and Wide, Proceedings of the 11th International Conference of AIDA, Bucharest, 2015, Bucharest, Editura UniversităȚii din București : 405-412.

Peña-Chocarro L., Pérez-Jordà G., Morales-Mateos J. \& Zapata L. 2015 - Storage in traditional farming communities of the western Mediterranean : Ethnographic, historical and archaeological data. Environmental Archaeology 20 (4) : 379-389. [En ligne] URL : http://www.tandfonline.com/ doi/abs/10.1179/1749631415Y.0000000004, DOI: 10.1179/1749631415Y.0000000004. Consulté le 15 juin 2017.

Prévost V. 2008 - Une tentative d'histoire de la ville ibadite de Sadrata. In : Transferts culturels dans le monde hispanique. Mélanges de la Casa Velasquez : 38-2

Renisio A. 1932 - Études sur les Dialectes berbères des Beni Iznassen, du Rif et des Senhadja du Srair Grammaire, textes et lexique. Paris. Ernest Leroux, 465 p.

Revel N. 1990 - Fleurs de paroles : histoire naturelle Palawan. Paris, Peeters-SELAF. [En ligne], URL :http://linguistics.oxfordre.com/view/10.1093/acrefore/9780199384655.001.0001/ acrefore-9780199384655-e-232?rskey=hOxCDP\&result=1. Consulté le 21 juillet 2017. 
Sánchez P. \& Vicente Á. 2012 - Variación dialectal en árabe marroquí: əl-haḍra š-šāmālīya u la-hḍ̣a l-maṛ̂ā̄ě̌ǐya. In : Barontini A., Pereira C., Vicente Á. \& Ziamari K. (Ed.) Dynamiques langagières en Arabophonies : variations, contacts, migrations et créations artistiques. Hommage offert à Dominique Caubet par ses élèves et collègues. Zaragoza, Universidad de Zaragoza : 223-252.

Vicente Á. 1998 - Un dialecte de type montagnard au Maroc : le parler d'Anjra. In : Aguadé J., Cressier P. \& Vicente Á. (Ed.) Peuplement et arabisation au Maghreb occidental, Madrid-Zaragoza, Casa de Velázquez-Universidad de Zaragoza : 121-130.

Vicente Á. 2000 - El dialecto árabe de Anjra (norte de Marruecos). Estudio lingüístico y textos. Zaragoza, Universidad de Zaragoza, 285 p.

Vicente Á. 2002 - El dialecto árabe de los Masmûda (norte de Marruecos). Estudios de dialectología norteafricana y andalusí $6: 221-231$.

Vicente Á. 2017 - Les parlers arabes montagnards du Nord du Maroc. Une question d'identité langagière. In : Vicente Á., Caubet D. \& Naciri-Azzouz A. (Ed.) La région du Nord-Ouest marocain: Parlers et pratiques sociales et culturelles. Zaragoza, Prensas de la Universidad de Zaragoza : 29-50.

Vicente Á., Caubet D. \& Naciri-Azzouz A. (Ed.). 2017 - La région du Nord-Ouest marocain : Parlers et pratiques sociales et culturelles. Zaragoza, Prensas de la Universidad de Zaragoza, $357 \mathrm{p}$.

Vignet-Zunz J. 2014 - Jbala du Rif. Des lettrés en montagne. Casablanca, La Croisée des Chemins IREMAM, $415 \mathrm{p}$

Ziamari K. \& Barontini A. 2008 - Quelques éléments de description d'un parler Jebli (Ourtzagh, Maroc). Estudios de dialectología norteafricana y andalusí 12 : 43-59.

Zohary D., Hopf M. \& Weiss E. 2012 - Domestication of plants in the old world, Oxford University Press, Oxford. 1406 (1581) 175 DOI: 10.1126/science.187.4174.319.

\section{NOTES}

1. Yildiz Aumeeruddy-Thomas, Dominique Caubet, Younes Hmimsa et Ángeles Vicente sont les co-éditeurs de ce numéro spécial. Yildiz Aumeeruddy-Thomas en a assuré la coordination principale.

2. Descriptions faites au début du xxe siècle (Colin 1921, Lévi-Provençal 1922), ou au début des années 90 par les étudiants de Lévy (voir Khoukh 1993, Maghdad 1993 et Abou El Haja 1995), par Messaoudi (1999) ; puis au début du XxI ${ }^{\mathrm{e}}$ siècle par Vicente $(1998,2000,2002)$, Sánchez \& Vicente 2012, par Ziamari et Barontini (2008) etc., et enfin par les jeunes chercheurs ayant publié dans l'ouvrage de Vicente et al. (2017) et qui ont enquêté en 2012 dans le cadre du PICS et du Programme du CJB.

\section{RÉSUMÉS}

Ce numéro spécial situé à la croisée d'approches interdisciplinaires ethnobotanique, linguistique et anthropologique, révèle les modalités d'interactions entre les hommes, la nature et autrui chez les Jbala et dans le Rif, au Maroc. Nous observons une diversité linguistique élevée, des pratiques 
anciennes et très résilientes sur la nature ainsi qu'une agrobiodiversité associée exceptionnelle. Nous suggérons que l'organisation sociale rifaine ainsi que la diversité des parlers constituent des éléments identitaires stables créant des frontières entre les différents groupes sociaux arabophones et berbérophones d'origines variées du Rif, et du Pré-Rif. Des échanges aux frontières dans les souks ou lors de mariages, contribuent à la richesse linguistique ainsi qu'à l'agrobiodiversité. Celle-ci repose, en outre, sur la biodiversité naturelle et sur des échanges historiques avec la Méditerranée. Ces travaux montrent également une grande proximité entre les habitants du Rif et la nature, y compris des relations sociales complexes avec des êtres intangibles. Ces collectifs hybrides, les dynamiques langagières, et la richesse des techniques et de l'agrobiodiversité associée subissent aujourd'hui des processus de nivellement liés à la globalisation des échanges.

This special issue, at the crossroads of ethnobotanical, linguistic and anthropological interdisciplinary approaches, reveals the modalities of interactions between men, nature and 'otherness' in the Jbala and the Rif regions, in Morocco. We observe great linguistic diversity, ancient and very resilient practices on nature, as well as exceptional agrobiodiversity. We suggest that the social organization in the Rif, as well as the diversity of dialects and languages, constitute stable identity elements of identity creating boundaries between the various Arabic and Berber-speaking social groups of various origins in the Rif and the Pre-Rif regions. Exchanges at the borders, in souks and through marriages, enhance linguistic richness as well as agrobiodiversity. The latter is also based on natural biodiversity and on historical exchanges with the Mediterranean area. The present issue also shows great proximity between the inhabitants of the Rif region and nature, including complex social relations with intangible beings. These hybrid collectives, the language dynamics, and the richness of techniques and of agrobiodiversity are now undergoing leveling processes linked to the globalization of trade.

\section{INDEX}

Mots-clés : interactions Culture-Nature, ethnobotanique et linguistique, frontières, échanges, nord du Maroc, Rif

Keywords : Culture-Nature interactions, ethnobotany and linguistics, frontiers, exchanges, northern Morocco, Rif

\section{AUTEURS}

\section{YILDIZ AUMEERUDDY-THOMAS}

Directeur de Recherche, CNRS, Centre d'Écologie Fonctionnelle et Évolutive (UMR5175 CEFE)

Campus CNRS, 1919 route de Mende - 34293 Montpellier cedex 5, France

yildiz.aumeeruddy-Thomas@cefe.cnrs.fr

\section{DOMINIQUE CAUBET}

Professeur émérite d'arabe maghrébin, INALCO. LaCNAD et Centre Jacques Berque; 16 Bd. A.

Blanqui, 75013 PARIS

caubet.dominique@yahoo.fr

\section{YOUNES HMIMSA}

Enseignant chercheur à l'Université Abdelmalek Essaâdi, Département des Sciences de la Vie (Biologiste et ethnobotaniste, Membre du laboratoire de Botanique appliquée (Equipe Bio- 
Agrodiversité)), Faculté Polydisciplinaire de Larache. B.P. 745, Poste Principale, Larache 92004 MAROC.

hmimsayounes@gmail.com

\section{ÁNGELES VICENTE}

Maître de conférences (HDR), Universidad de Zaragoza, mavicen@unizar.es

Calle Pedro Cerbuna, 12

Facultad de Filosofía y Letras - Universidad de Zaragoza

50009 Zaragoza - Espagne 